\title{
Taking Times Out: Tense Logic as a Theory of Time ${ }^{1}$
}

\author{
Thomas Pashby ${ }^{\mathrm{a}}$ \\ ${ }^{a}$ Mudd Hall of Philosophy, 3709 Trousdale Parkway, University of Southern California, Los \\ Angeles, CA 90089, USA.
}

Ulrich Meyer's fascinating book The Nature of Time uses a novel development of Arthur Prior's tense logic to argue for a 'modal' account of time. Meyer's metaphysical view requires tense primitivism (the view that truths are irreducibly tensed) and so, in Quine's turn of phrase, trades ontology for ideology. Meyer's temporal metaphysics eschews ontological commitment to temporal instants through the introduction of so-called 'ersatz times,' developed in analogy with the ersatz possible worlds of modal logic.

In modal logic, linguistic ersatzism about possible worlds is associated with actualism (the thesis that only actual objects and events exist) so one might expect an advocate of ersatz times to endorse presentism (the thesis that only present objects and events exist). Many philosophers of physics (myself included) take presentism to be inconsistent with special relativity. Meyer, however, argues that the analogy between presentism and actualism is weak, and denies the presentist's claim that the present is metaphysical privileged. Furthermore, Meyer claims that relativity allows for the introduction of 'hybrid views' that combine distinct, seemingly incompatible metaphysical accounts of time and space. In particular, Meyer advocates a metaphysics of relativistic spacetime that combines spatial substantivalism with his 'modal' view of time.

The argument of the book follows an almost Aristotelian methodology. First, in Chapter 1, the desiderata for a theory of time are outlined. Next, rival metaphysical theories are dismissed one by one: varieties of temporal relationalism in Chapter 2, and temporal substantivalism in Chapter 3. Then the preferred theory is outlined (in Chapters 4,6 and 8) and declared the victor, with the rest of the book devoted to showing how remaining difficulties may be accommodated. In particular, there are chapters that deal with temporal propositions and truthmakers (Chapter 5), temporal structure (Chapter 7), presentism (Chapter 9), becoming (Chapter 10), motion (Chapter 11) and relativity theory (Chapter $12)$.

\footnotetext{
${ }^{1}$ This article was written with the financial assistance of a Provost's Postdoc in the Humanities from the University of Southern California. Thanks to Andrew Bacon, Steven Savitt, and the South California Philosophy of Physics Group, particularly Craig Callender and David Malament, for useful comments and conversations.
} 
There is much to admire about Meyer's book: aside from the novel metaphysical view advocated within, this elegantly slim volume contains authoritative discussions of - if not all then an immodest subset of - the key issues in contemporary philosophy of time. I will, however, take issue with two claims in particular: first, that Meyer's novel argument against temporal relationism rules out Russell's theory of time; it does not. Second, that hybrid views provide a sensible and robust approach to the metaphysics of time in relativity theory. Before that, let's delve into some of the details of Meyer's theory of time.

Meyer, who rejects substantial times (as exemplified by Newton's Absolute Time) as "ontologically extravagant" (p. 28), aims to give an account of instants of time in terms of sets of sentences of tense logic, that is ersatz times. In so doing, Meyer hopes to limit his metaphysical commitments to just the tense operators and axioms of a minimal tense logic - to temporal ideology alone. This trading of ontology for ideology is, for Meyer, the main attraction of his view:

The advantage of the times-as-sets view is that it preserves the ontological frugality of tense primitivism. It provides the needed referents for dates without incurring commitment to temporal propositions, temporal parts, metaphysically basic time points, or any other recognizably temporal entities. (p. 66)

However, nowhere does Meyer explain why it is such a good idea to eschew any recognizable temporal ontology, other than the vague claim that "[t]heories with fewer ideological and ontological commitments are usually thought to be preferable" (p. 3) - by whom, and why?

The closest Meyer comes to an official answer (in Chapter 1) is that the best theory of time will (i) solve the Problem of Change, (ii) mesh well with physics, and (iii) accord with ordinary ways of speaking about time, all with a minimum of ontological and ideological commitments. In reverse order: By design, a 'modal' theory of time scores highly when it comes to modeling our ordinary tensed temporal discourse. However, despite his best efforts, I will argue that Meyer's project does not mesh as well with spacetime physics as he would have us believe. Finally, the Problem of Change

is the task reconciling the possibility of change with the Indiscernability of Identicals. An object $[a]$ experiences change just in case it possesses some property $K$ at one time that it lacks at another. (p. $4)$.

That is, $a$ experiences change if and only if the following is true: $a$ is $K$ and $a$ is not $K$. This apparent contradiction must be avoided by a theory of time.

Equipped with an ontology of substantial times the resolution of the paradox is clear: $a$ is $K$ at time $t$ and $a$ is not $K$ at time $t^{\prime}$, with $t \neq t^{\prime}$. However, in Chapter 3, Meyer argues that things are not so simple and the introduction of substantial times causes more problems than it solves. Meyer's 'modal' theory approaches the problem differently. Taking his cue from natural language, in which 
we cannot attribute a property to an object without committing ourselves, by our choice of tense, to a past, present, or future time at which the object possesses that attribute[,] (Meyer, 2013, p. 37)

Meyer endorses the property-time link - the idea that truths about objects come irreducibly tensed.

The core idea of Meyer's project is that, by taking objects and tensed property attributions as fundamental, the property-time link allows him to reconstruct the times needed to solve the Problem of Change without a commitment to an ontology of times. Instead, Meyer's view of time relies on a timeless ontology of objects and properties, ${ }^{2}$ from which a non-trivial ideological commitment to tense logic allows him reconstruct an ersatz time series of 'possible presents.' To see how this works we need some details of tense logic.

Tense logic is nothing new, and it was originally introduced by Prior (1957) through an analogy with modal logic (hence the idea of a 'modal' theory of time). Taking the models as our starting point, a possible world semantics for modal logic builds on propositional logic in the following way. We begin with a set of atomic sentences $A, B, C, \ldots$ of a formal language $\mathcal{L}$. A model for propositional logic is a valuation, $v$, which assigns either 'true', $v(\varphi)=T$, or 'false,' $v(\varphi)=F$, to every sentence of the language, $\varphi \in \mathcal{L}$. Modal logic introduces a set of worlds, $\mathfrak{W}$, each of which is a model of propositional logic. Tense logic replaces this set of worlds with a set of times, $\mathfrak{T}$, each of which corresponds to a model of propositional logic in the same way. That is, each time $t \in \mathfrak{T}$ is a valuation, $v_{t}$, of the language $\mathcal{L}$.

Aside from the usual logical connectives 'not' $(\neg)$ and 'implies' $(\rightarrow)$, modal logic introduces a necessity operator $(\square)$ and a possibility operator $(\diamond)$. In tense logic, these operators have an interpretation in terms of times: necessary means true at all times and possible means true at some time. However, tense logic aims to model tensed temporal discourse so is based instead on two 'possibility' operators which distinguish between past and future: 'It was the case that' $(P)$ and 'It will be the case that' (F). As a result, tense logic comes equipped with two 'dual' operators that play the role of necessity: $\mathrm{H}:=\neg \mathrm{P} \neg$ and $\mathrm{G}:=\neg \mathrm{F} \neg$.

A model for Meyer's minimal tense logic $Z$ (for Zeitlogik) is a triple $\langle\mathfrak{T},<$ $, p\rangle$, where $\mathfrak{T}$ is a set of time-indexed models of propositional logic, $<$ is a relation between times (the 'earlier-than' relation), and $p \in \mathfrak{T}$ is a particular time chosen as 'the present' (in modal logic, this would be a possible world chosen as 'actual'). A sentence $\varphi$ is true at a time $t$ iff $v_{t}(\varphi)=T$. We can now give a semantics for these tense operators as follows:

$\mathrm{P} \varphi$ is true at $t$ iff $\exists t^{\prime}$ such that $t^{\prime}<t$ and $v_{t^{\prime}}(\varphi)=T$.

$\mathrm{H} \varphi$ is true at $t$ iff $\forall t^{\prime}$ such that $t^{\prime}<t, v_{t^{\prime}}(\varphi)=T$.

$\mathrm{F} \varphi$ is true at $t$ iff $\exists t^{\prime}$ such that $t<t^{\prime}$ and $v_{t^{\prime}}(\varphi)=T$.

$\mathrm{G} \varphi$ is true at $t$ iff $\forall t^{\prime}$ such that $t<t^{\prime}, v_{t^{\prime}}(\varphi)=T$.

${ }^{2}$ This is made quite explicit in Meyer's development of a quantified tense logic (in Chapter 8 ) in terms of a tenseless domain of objects. 
Furthermore, a sentence $\varphi$ is true simpliciter iff it is true at the present time $p$.

A few remarks here will serve to introduce the details of Meyer's project. First, the interpretation of the tense operators above introduces an explicit quantification over times, which by Quineian strictures incurs an ontological commitment. It is precisely this ontological commitment that Meyer hopes to avoid through (i) the replacement of times by ersatz times and (ii) a commitment to tense primitivism, the idea that tense operators are conceptually basic. In sum, Meyer wants $P$ and $F$ left uninterpreted so that he can build up an interpretation of the time series (given by ersatz times) in terms of $\mathrm{P}$ and $\mathrm{F}$, rather than vice versa. Having done so, he introduces an object language that allows for quantification over the ersatz time series, which allows $\mathrm{P}$ and $\mathrm{F}$ to be interpreted as above, replacing times with ersatz times. In this way, he avoids ontological commitment to substantial temporal instants.

Second, Meyer's commitment to a minimal tense logic takes pains to avoid making any particular assumptions about the (ersatz) time series. In reading the interpretation above it would be easy to assume that the times $\mathfrak{T}$ are ordered by $<$ as if they were elements of the real line, but this is far from necessary: models of $Z$ exist in which the time series branches (in which case the relation $<$ is not comparable), or has endpoints, or is not dense, or is discrete. Indeed, the relation $<$ need not even form a (strict) preorder on $\mathfrak{T}$ since models of $Z$ exist in which it is not transitive. Each additional condition on the time series corresponds to an additional axiom of the tense logic. By restricting his ideological commitments to just the minimal axiom set $Z$, Meyer hopes to secure a priori status for our knowledge of the nature of time and apparently he regards the theorems of $Z$ as analytic truths, i.e. true by definition (pp. 47-48).

Third, philosophical criticism of tense logic has often involved showing that it is not fit for (its original) purpose as a formal account of the uses of verb tense in natural language. By presenting sentences in English that cannot be expressed in tense logic without the introduction of further operators (such as Kamp's 'A child was born which would be king') it has been argued that a sufficiently expressive tense logic must ultimately rely on quantification over times (Massey, 1969; van Benthem, 1983). In essence, the complaint is that tense logic is a mere notational variant of a classical temporal logic that merrily quantifies over an ontology of times. Meyer's answer to this is, again, the introduction of quantification over an ersatz times series, which allows for any such sentence to be meaningfully expressed without quantification over (substantial) times and without the introduction of further tense operators. ${ }^{3}$

So the idea of an ersatz time series is crucial to Meyer's positive project, which he calls linguistic ersatzism about time (in analogy with ersatzism about possible worlds). Meyer defines a possible present $\mathfrak{p}$ as a maximal consistent sets of sentences ${ }^{4}$ of the tense logic $Z$ (which includes negation $(\neg)$ and ma-

\footnotetext{
${ }^{3}$ However, as Meyer (p. 65) notes, the quantification over ersatz times cannot be eliminated to produce a sentence involving just $\mathrm{P}$ and $\mathrm{F}$.

${ }^{4} \mathrm{~A}$ set of sentences is maximal if it contains either $\varphi$ or $\neg \varphi$ for every sentence in the
} 
terial implication $(\rightarrow)$, as well as $\mathrm{P}$ and $\mathrm{F}$ ). Choose some possible present $\mathfrak{p}=\{\mathrm{P} \varphi, \mathrm{F} \varphi, \mathrm{FP} \varphi, \ldots\}$ to be the actual present. Because the set of sentences is maximal consistent, $\mathfrak{p}$ provides plenty of information about the past and future as well as the present. The trick is to use that information to define an entire (ersatz) time series.

To avoid reference to times, Meyer (p. 61) now defines 'true at' in terms of sentences:

A sentence $\varphi$ is true at a set of sentences $S$ iff $\varphi \in S$.

This provides an interpretation of 'earlier-than' and 'later-than' in terms of the tense operators rather than the other way around:

$$
\begin{aligned}
& S^{\prime}<S \text { iff } \mathrm{F} \varphi \in S^{\prime} \text { whenever } \varphi \in S . \\
& S^{\prime}>S \text { iff } \mathrm{P} \varphi \in S^{\prime} \text { whenever } \varphi \in S .
\end{aligned}
$$

Remarkably, Meyer (2009) proves that whenever a possible present $\mathfrak{p}$ contains a past (resp. future) tense sentence $\mathrm{P} \varphi(\mathrm{F} \varphi)$ there exists a possible present $\mathfrak{t}$ such that $\varphi \in \mathfrak{t}$ and $\mathfrak{t}<\mathfrak{p}(\mathfrak{t}>\mathfrak{p})$.

Any claim made by a possible present regarding the past or future, then, is backed up by the existence of a possible present at which that claim is true. To construct an ersatz time series we take these possible presents, nested inside $\mathfrak{p}$ like Russian dolls, and string them together one after the other. More formally, each possible present $\mathfrak{p}$ defines a ersatz time series $\mathcal{T}_{\mathfrak{p}}$ which includes all and only those possible presents which can be reached from $\mathfrak{p}$ via a connected string of possible presents. Crucially, if $\mathfrak{q}$ is another possible present such that $\mathfrak{q} \in \mathcal{T}_{\mathfrak{p}}$ then $\mathcal{T}_{\mathfrak{q}}=\mathcal{T}_{\mathfrak{p}}$. Furthermore, each possible present $\mathfrak{p}$ defines a model of tense $\operatorname{logic}\left\langle\mathfrak{T}_{\mathfrak{p}},<, \mathfrak{p}\right\rangle$ in which all and only the sentences of $\mathfrak{p}$ are true, and conversely each model of tense logic defines a possible present (Meyer, 2013, p. 62).

This reconstruction of a time series from sentences of tense logic forms the core of Meyer's positive project, which he takes to be a theory of time on a par with Russell's relationist theory of time. The contrast between the two is instructive since both aim to avoid commitment to instants of time by means of logical constructions. Russell (1936), inspired by A. N. Whitehead, attempted a reconstruction of the time series (i.e., a set of temporal instants structured like the points of the real line) from a class of temporally extended events with two relations defined on them: overlap ( $\circ$ ) and complete temporal precedence $(\ll)$.

Regardless of the success of this project (the history of which is recounted by Meyer, pp. 8-13), its ontology is clear: there exists a class of events and relations on those events, and time is nothing but an ordered collection of classes of events. The contrast between Russell's relational theory of time and Meyer's 'modal' theory of time can be drawn in two directions. In terms of ontology: Russell relies on the existence of events and their relations; Meyer on the existence

language $\varphi \in \mathcal{L}$. 
of objects and their properties. In terms of ideology: Russell eschews tense altogether; Meyer relies on it.

One respect in which Meyer thinks he has the advantage is that the relationist must assume that the actual course of events has particular structural characteristics in order to construct a time series, whereas the minimal tense logic $Z$ makes no assumptions about whether the time series is discrete or continuous, is linear or branches, etc. It seems to me, however, that the relationist is free to pursue alternative schemata in the same manner, viz., as ways that the time series could have been, but isn't. Just as Meyer's ideological commitment is held fixed between these possible (ersatz) time series, so too could the relationist's ontological commitments be maintained while exploring alternative possible event structures.

In terms of overall metaphysical parsimony, then, it is hard to see which has the advantage, and it seems that Meyer finds temporal relationism quite attractive; certainly he prefers it to substantivalism. In the book, what settles the case in favour of Meyer's theory is a new argument against relationism. But this argument is far from the knock-out blow that Meyer takes it to be.

The argument begins with a discussion of the difficulties in maintaining that events can be both metaphysically complex and the primary occupants of the time series. Essentially, the worry is that if events are defined in terms of their time of occurrence or spatio-temporal location then they cannot serve as the ingredients of the relationist's recipe for constructing times. This difficulty was resolved by Russell in taking events and their temporal relations to be metaphysically basic (see Meyer, pp. 14-17). The further difficulty raised by Meyer is that the temporal relationist still owes us an account of the relationship between objects and events. Here's the difficulty, as he sees it:

If events are the primary occupants of the time series then material objects relate to times only indirectly, by participating in events that stand in temporal relations to one another. (p. 17, original emphasis)

Meyer argues that in attempting to give any account of participation (i.e. the supposed relation that objects bear to events) the temporal relationist encounters what he claims is a fatal dilemma: either the account is so restrictive as to rule out participation tout court, or it involves treating events as metaphysically complex. Since the temporal relationist cannot take the second horn, she must take the first. But then, complains Meyer,

we have nothing plausible to say about how ordinary material objects relate to time. And if we have no account of the relation of material objects and time then we have no account of what it is for an object to have a property at a time, and thus no solution to the Problem of Change. [...] Event-relationism about time is untenable. (pp. 19-20)

This conclusion, however, only follows if we assume that there is indeed a relation of participation connecting objects and events of which the temporal 
relationist owes us an account. If, for example, our relationist denied the independent existence of material objects by maintaining a strict event ontology then there could be no expectation of giving an account of participation in terms other than events and their relations. Furthermore, when it comes to ontological parsimony, a temporal relationist who is also an event ontologist would have an advantage over the event-relationist that Meyer considers.

But this event ontologist is far from hypothetical: this was precisely Russell's position during the period we are interested in here. From 1927 onward, Russell adopted an event ontology, according to which objects can be analyzed without remainder into events. That is, in Russell's event ontology, 'material objects'understood as comprising a metaphysical category distinct from events - do not exist.

In the same way that the temporal relationist proposes a reductive analysis of times in terms of inter-related events, so the event ontologist proposes that material objects - electrons, protons, atoms and the like - are nothing but collections of inter-related events. Regardless of whether or not the reduction was successful, there can be little doubt that this was Russell's intention:

Electrons and protons $[\ldots]$ are not the stuff of the physical world: they are elaborate structures composed of events, and ultimately of particulars. (Russell, 1927, p. 386).

Russell's reductionist (or eliminativist) intent regarding persisting objects is made particularly clear in his critical discussion of Whitehead (1925), who views spatio-temporally located events as mere aspects of a single persisting thing. ${ }^{5}$ Russell objects to this view "on purely logical grounds" as follows:

Given a group of events, the evidence that they are "aspects" of one "thing" must be inductive evidence, derived from perception [...] in calling two events "aspects" of one "thing," we imply that their likeness is more important than their difference; but for science both are facts, and of exactly the same importance. ${ }^{6}$ (Russell, 1927, p. 248)

The idea appears to be this: the series of events that seem to us to compose a single material thing are nevertheless numerically distinct and so cannot be regarded as the aspects of the same thing, on pain of logical contradiction. This is, in effect, to take the Problem of Change as an argument against the claim there exist persisting material objects, self-identical at every time they exist.

An event ontologist can hardly be expected to give an account of how nonexistent persisting material objects can come to possess contrary properties at distinct times. However, Meyer may still object that this is no solution to the

\footnotetext{
${ }^{5}$ I include this discussion in particular because Whitehead is cited by Meyer (p. 17) as a temporal relationist who introduces a notion of participation (called 'situation' by Whitehead).

${ }^{6}$ See also Russell's discussion of Whitehead in relation to the objects of perception (pp. 144-152).
} 
Problem of Change. But since Russell identifies what we would think of as a material object - a "thing" - with a series of events the solution is straightforward: $a$ is nothing but a series of events (i.e. concrete particulars of limited spatial and temporal extent) some of which are $K$ and some of which are not $K{ }^{7}$ There is no potential for logical contradiction lurking here: these events are numerically distinct and the Indiscernability of Identicals simply does not apply.

At best, Meyer's argument establishes that the temporal relationist must be a strict event ontologist. But since the relationist already accepts events into her ontology, what is the cost? Even by Meyer's own criteria things are looking quite good: in terms of physics, Russell's (1927) event ontology was motivated by consideration of general relativity; and Donald Davidson, the most prominent event ontologist of recent times, used event predication to give an account of adverbial modification in natural language (Davidson, 1980). At most, Meyer could complain about the lack of reference to "ordinary material objects," and the promissory status of a reduction of those objects and their spacetime locations to events and their relations - far from a knock-down refutation.

But Meyer's own ontology, and the resulting account of the nature of time, is hard to grasp. What exists, according to Meyer's metaphysics (as outlined in Chapter 8), is what lies in the domain of a quantified tense logic from which times have been eliminated in favour of ersatz times. Now I don't mean to suggest that this metaphysic is incoherent so much as vastly underspecified. For example, it is compatible with both substantivialism about space (according to which spatial points exist and material objects occupy those points) and relationism about space (according to which only material objects exist and space is to be given in terms of their relative positions).

Meyer rejects spatial relationism in favor of spatial substantivalism (in Chapter 3) but fails to consider the relationist re-casting of classical physics by Barbour and Bertotti (1982), which provides the means to resist Meyer's arguments in favour of substantial points. As detailed consideration of Barbourian physics shows (Belot, 1999; Pooley, 2001; Pooley and Brown, 2002), there is no explanatory gap here regarding inertial effects (which are accommodated along Machian lines), nor special difficulties concerning classical fields (which can be recast in terms of relationally specified field configurations).

Given Meyer's rejection of both temporal ontology and intrinsic metrical structure (on p. 75), this strikes me as a missed opportunity: the latter of these corresponds to what Barbour (1982, p. 260) calls the Second Mach's Principle, and there is a close resemblance of Meyer's possible presents to Barbour's 'time capsules.' Barbour (1999) defines a time capsule as a "static configuration that appears to contain mutually consistent records of processes that took place in a past in accordance with certain laws" (p. 31). This leads him to hope that

all appearances of time will have been reduced to a common basis:

\footnotetext{
${ }^{7}$ This is essentially Russell's definition of motion (Russell, 1927, p. 246).
} 
special structure in individual Nows. [...] The inference that [the time line] exists can emerge from a single Now. The instant is not in time - time is in the instant. (Barbour, 1999, p. 34)

As Ismael reflects:

Moments, in this picture, are elements of a grand configuration space, like the worlds in Lewis' modal universe; there is no timelike dimension constituted by them collectively and misrepresented by them individually. (Ismael, 2002, p. 319, original emphasis)

This seems like a pretty fair description of Meyer's possible presents too: mere descriptions of a momentary state of affairs containing information about the past, connected together by logical inference rather than temporal passage. And so it seems to me that, ultimately, Meyer is to be read as denying the reality of time in an analogous way to Barbour. This is the real reason why an accusation of presentism would be wide of the mark: Meyer doesn't think that time exists at all.

However, as Meyer simply fails to consider Barbour's relationist physics I cannot say how closely he takes his elimination of temporal ontology to approach Barbour's denial of time, or, indeed, similar positions motived by alternative formalisms of general relativity (Earman, 2002; Rovelli, 2009). Instead, when Meyer comes to consider relativistic spacetime he does so equipped with a commitment to spatial substantivalism and so an ontology of eternally existing spatial points. He also assumes a curiously anachronistic attitude towards the importance of the "reference frame," which "can be regarded as pair $R=\left\langle R_{s}, R_{t}\right\rangle$, where $R_{s}$ encodes the prescriptions for assigning spatial coordinates and $R_{t}$ those for temporal coordinates" (p. 114).

Meyer claims that the argument from accepting relativistic physics to accepting the metaphysical unity of space and time - the "inseparability argument" - is inconclusive. Instead he claims that there is no reason why one cannot combine substantivalism about space with relationism about time, or with Meyer's own view: "Tense operators," he says, "are devices for drawing simple temporal coordinate lines across space" (p. 129). The coordinate lines they draw depend on the reference frame, and each choice of reference frame leads to an alternative ersatz time series. Special relativity is to be respected by the proscription that no particular reference frame can be privileged over another.

One of the strange aspects of this view is the idea that a choice of reference frame - an assignment of spatial and temporal coordinates - is required to make sense of relativistic spacetime. Aside from the availability of coordinate independent descriptions (which Meyer acknowledges and claims to be able to recover) there is the fact that the existence of a global coordinate chart is neither sufficient to support a (frame dependent) split of spacetime into space and time, nor is such a split necessary to apply relativistic physics. In support of the latter claim, consider the 'GPS observables' of Rovelli (2002), which have an interpretation in terms of the position of four material points (i.e. GPS satellites) and do not correspond to a foliation of spacetime into space and time (i.e. a global time 
function). In support of the former, there exists a global coordinate chart for Gödel spacetime - a solution of Einstein's Equations with particularly strange temporal properties - despite the fact it has no closed spacelike hypersurfaces (Malament, 2012, p. 196).

I will return to Meyer's difficulties with Gödel spacetime in a moment, but there is also cause for skepticism that Meyer's reconstruction can be carried out at all, even in the simplest of cases. Take non-relativistic Galilean (or Neo-Newtonian) spacetime, which comes with a specification of a set of inertial frames and a uniquely specified set of instantaneous spatial 'slices' but without any particular inertial frame being privileged. In this case, Meyer's hybrid view says that each inertial (i.e. reference) frame describes the spatio-temporal paths of a set of persisting material objects - substantial spatial points. According to each of these frames, then, each of these 'particles of space' is at rest.

In that case, however, Meyer's official procedure for reconstructing an ersatz time series with non-denumerably many instants will not work. Earlier in the book, Meyer (p. 68) considers a (non-stationary) point particle following a continuous curve. By referring to the points of space that are occupied by the particle at different points on the curve we can distinguish (ersatz) times. Meyer (2009) shows that even if the formal language used to represent tensed facts has a countable vocabulary that one can distinguish continuously many times (by making use of Dedekind cuts). But what if the path of the particle is stationary?

Consider a free particle with zero momentum and initial position $a$. The path of the particle is given by a parameterized curve $f(t)=a$, for all $t$. Let $L a$ be the proposition that the particle is located at $a$. How many ersatz times are there? At first sight, it seems as if there is one: the time at which $L a$ is true. However, Meyer (p. 68) does have another trick up his sleeve: a possible present $\mathfrak{p}=\{L a, \mathrm{P} L a, \operatorname{PP} L a, \operatorname{PPP} L a, \ldots\}$ corresponds to an ersatz time series with at least four distinct times, and one could keep on going. But there is no way to extend this time series to a non-denumerable infinity of times by using Meyer's original construction, which relies on a non-denumerable infinity of positions to escape the limitation of the language to merely countably many names. Therefore, Meyer's claim to be able to recover a description of an empty spacetime by means of an ersatz time series remains unsupported. ${ }^{8}$

There are also difficulties with the other hybrid view explicitly discussed by Meyer: the combination of temporal relationism with spatial substantivalism in special relativity. As Meyer (p. 128) points out, the posits of Russell's relational theory of time are incompatible with Robb's relativistically invariant reading of temporal precedence $(\ll)$ in special relativity. Meyer's suggestion is that the temporal relationalist should adopt a frame-relative version of temporal

\footnotetext{
${ }^{8}$ I also share Savitt's (forthcoming) concern that a supposed 'reconstruction' of Minkowski spacetime from a set of reference frames and an equivalence relation on them encoding the symmetry group of the spacetime cannot bear the metaphysical weight attached to it by Meyer.
} 
precedence, thus attributing contrary temporal relations to spacelike separated pairs of events (with respect to some pair of inertial frames). But to do so would betray the motivation of the project, which was to reconstruct times from an ontology of events and their temporal relations: a temporal relation among events is a two-place relation; it is not a three-place relation between two events and an arbitrarily chosen reference frame.

This sort of concern may even lead one to be skeptical of Meyer's overall project. Meyer introduces his book as providing an answer to the question: "What sort of things are instants of time?" (p. 1). But, according to a significant (but, perhaps, minority) view among philosophers of physics, special relativity teaches us that there simply are no instants of time. That is, there is no such thing as a spatially extended temporal instant composed of coexistent events. What underlies this claim is the idea that events are partially ordered by Robb's temporal precedence relation, not totally ordered. As Wüthrich (2010) puts it:

Special-relativistic theories admit only a partial temporal ordering of events. The loss of absolute simultaneity leads to a loss of comparability: with an interpretation of the binary ordering relation as "being earlier than or simultaneous to" - it is a temporal ordering that we are seeking after all-pairs of spacelike related events do not stand in this relation. (p. 260, original emphasis)

So, rather than dropping Robb's invariant relation, we should abandon the idea that pairs of spacelike related events can be temporally ordered, and with it the notion of a spatially extended instant of time.

Finally, concerning the extension of Meyer's hybrid views to general relativistic spacetimes, his success relies on the availability of a (not necessarily unique) decomposition into time and space. And, what is more, this split must suffice for an ontological interpretation in terms of a set of substantial spatial points which persist through time. It seems to me that this must correspond to a foliation of spacetime into Cauchy surfaces (spatial hypersurfaces pierced exactly once by each inextensible causal curve). Formally, global hyperbolicity provides a necessary and sufficient condition for the existence of a Cauchy time function, i.e., a foliation of a relativistic spacetime $\mathcal{M}$ into Cauchy surfaces $S$ with the structure $\mathbb{R} \times S$ (Minguzzi and Sánchez, 2008, Thm. 3.75). But global hyperbolicity lies to the strong end of causal conditions for relativistic spacetimes. At the other end lies Gödel spacetime.

Meyer (p. 123) admits that the existence of Gödel spacetime as a solution of Einstein's Equations provides strong support for the inseparability argument in General Relativity. I am not convinced by his response. If Gödel spacetime is to be admitted as a genuine physical possibility then, Meyer says,

there might only be a local way of understanding tensed sentences, and thus only a local way of abstracting times from them. In such a case, there would be something irreducibly 'patchy' about the temporal aspect of reality. (Meyer, 2013, p. 131) 
Presumably Meyer means to say that, although in this case there will be no genuine hybrid view available, if spatially extended instants of time can be defined locally then the same construction of ersatz times can be applied and that suffices to support his a priori commitment to the tense $\operatorname{logic} Z$ as a theory of time.

Despite the existence of closed timelike curves through every point of Gödel spacetime, there are indeed no closed timelike nor null geodesics (Malament, 2012, p. 209). An observer following a timelike geodesic might hope, therefore, to construct a local time series that extends at least somewhat beyond her onedimensional path through spacetime. She may have some limited success, but if she attempts to follow Einstein's clock synchronization protocol she will find that light rays will not travel beyond the critical radius $r_{c}$ and, indeed, any light ray she sends out will come back to meet her an indefinite number of times (the 'Boomerang Effect') (Malament, 2012, p. 207). ${ }^{9}$

More to the point, Meyer's commitment to the tense logic $Z$ as an a priori theory of time makes him particularly vulnerable to a close relative of Gödel's argument in favor of the ideality of time. Earman's (1995) careful analysis of Gödel's argument makes it clear that Gödel is missing an implicit premise that would get him to the conclusion that time in our world is ideal. Earman's first try at the missing premise is this:

(P7.1) The existence of an objective lapse is not a property that time can possess contingently. (Earman, 1995, p. 197)

If this premise is true then Gödel's argument succeeds. Earman is not convinced by the plausibility of this premise as he does not share the underlying intuition that one can arrive at such truths "just by reflecting on the concept of time" (Earman, 1995, p. 198). Meyer apparently does, however.

Recall that Meyer, in restricting his commitments to a minimal tense logic, refuses to take a stand on whether the time series is closed or open. ${ }^{10}$ But here we are not talking of 'the' time series - Gödel spacetime presents a situation in which that notion makes no sense at all. We must, therefore, restrict our attention to local time series, given by timelike curves. Since these curves can be either open or closed, such facts about the topology of time will be relative to one's particular path through the universe. There is, then, no knowledge to be had about 'time' considered separately from spacetime. ${ }^{11}$

If our knowledge of time is to be analytic a priori, as Meyer would like, then it is a matter of metaphysical necessity. And if we allow that General Relativity and Einstein's Equations provide a valid notion of physical possibility then the possible universes they describe they must conform to those necessary

\footnotetext{
${ }^{9}$ Rindler's (1981) distinction between 'public' and 'private' spaces in cosmological models also seems relevant here. This is discussed (in another context) by Malament (2012, pp. 163-164).

${ }^{10}$ See for discussion of this issue (Meyer, 2013, pp. 45-47, 69-71).

${ }^{11}$ A related point is made by Earman (1972, p. 82) against the causal theories of time of Grünbaum and van Fraassen.
} 
truths. That is, since Gödel spacetime is a solution of Einstein's Equations, it must conform to any and all metaphysical necessities. Contrapositively, if some purported metaphysical necessity fails to hold in Gödel spacetime then it is no metaphysical necessity at all. The dilemma faced by Meyer is as follows: either the interconnection of time and space in Gödel spacetime holds necessarily, in which case the inseparability argument goes through, or the separation of time and space is a contingent matter, in which case there is little scope for necessary truths about time alone.

In conclusion, I commend Meyer's book as both a very readable, albeit opinionated, introduction to the philosophy of time and an exemplar of the best work in the field. However, Meyer's hope to have given a metaphysical theory of time that floats free of contingent matters of fact (and, indeed, physics) sits uncomfortably with the idea that relativity theory provides valuable insights into the nature of time, and the details of his metaphysic remain to be fleshed out. More generally, it seems to me that analytic metaphysics still has much to gain by adopting Quine's naturalistic attitude of 'no first philosophy' and so, when it comes to understanding the nature of time, looking first to physics rather than language or intuitions.

Barbour, J., 1999. The End of Time. New York: Oxford University Press.

Barbour, J. B., 1982. Relational concepts of space and time. British Journal for the Philosophy of Science 33 (3), 251-274.

Barbour, J. B., Bertotti, B., 1982. Mach's principle and the structure of dynamical theories. Proceedings of the Royal Society of London A. Mathematical and Physical Sciences 382 (1783), 295-306.

Belot, G., 1999. Rehabilitating relationalism. International Studies in the Philosophy of Science 13 (1), 35-52.

Davidson, D., 1980. Essays on Actions and Events. Clarendon Press.

Earman, J., 1972. Notes on the causal theory of time. Synthese 24 (1-2), 74-86.

Earman, J., 1995. Bangs, Crunches, Whimpers and Shrieks. Oxford: Oxford University Press.

Earman, J., 2002. Thoroughly modern McTaggart or what McTaggart would have said if he had read the general theory of relativity. Philosophers' Imprint $2(3), 1-28$.

Ismael, J., 2002. Rememberances, mementos, and time-capsules. In: Callender, C. (Ed.), Time, reality and experience. No. 50. Cambridge University Press, pp. $317-329$.

Malament, D. B., 2012. Topics in the foundations of general relativity and Newtonian gravitation theory. University of Chicago Press.

Massey, G. J., 1969. Tense logic! why bother? Noûs, 17-32. 
Meyer, U., 2009. Times in tense logic. Notre Dame Journal of Formal Logic 50 (2), 201-219.

Meyer, U., 2013. The Nature of Time. Oxford University Press.

Minguzzi, E., Sánchez, M., 2008. The causal hierarchy of spacetimes. Recent developments in pseudo-Riemannian geometry, ESI Lectures Mathematical Physics, 299-358.

Pooley, O., March 2001. Relationism rehabilitated? II: Relativity. URL http://philsci-archive.pitt.edu/221/

Pooley, O., Brown, H. R., 2002. Relationalism rehabilitated? I: Classical mechanics. The British Journal for the Philosophy of Science 53 (2), 183-204.

Prior, A. N., 1957. Time and Modality. Oxford: Oxford University Press.

Rindler, W., 1981. Public and private space curvature in robertson-walker universes. General Relativity and Gravitation 13 (5), 457-461.

Rovelli, C., Jan. 2002. GPS observables in general relativity. Physical Review D 65 (4), 044017+.

Rovelli, C., 2009. Forget Time, FQXi Essay Competition. URL http://arxiv.org/abs/0903.3832v3

Russell, B., 1927. The Analysis of Matter. Cambridge: Routledge.

Russell, B., 1936. On order in time. In: Proceedings of the Cambridge Philosophical Society. Vol. 32. Cambridge University Press, pp. 216-228.

Savitt, S., forthcoming. Review of The Nature of Time. Mind.

van Benthem, J. F. A. K., 1983. The Logic of Time. Dordrecht: Reidel.

Whitehead, A. N., 1925. Science and the modern world. Cambridge University Press.

Wüthrich, C., 2010. No presentism in quantum gravity. In: Petkov, V. (Ed.), Space, Time, and Spacetime: Physical and Philosophical Implications of Minkowski's Unification of Space and Time. Vol. 167 of Fundamental Theories of Physics. Springer, pp. 257-280. 